\title{
Heavy quark energy loss in the quark-gluon plasma in the Moller theory
}

\author{
B. Blok ${ }^{1, a}$ \\ ${ }^{1}$ Department of Physics, Technion-Israel Institute of Technology, Haifa, Israel
}

Received: 29 February 2020 / Accepted: 29 July 2020 / Published online: 12 August 2020

(C) The Author(s) 2020

\begin{abstract}
We study the energy loss of a heavy quark propagating in the quark-gluon plasma (QGP) in the framework of the Moller theory, including possible large Coulomb logarithms as a perturbation to BDMPSZ bremsstrahlung, described in the harmonic oscillator (HO) approximation. We derive the analytical expression that describes the energy loss in the entire emitted gluon frequency region. In the small frequencies region, for angles larger than the dead cone angle, the energy loss is controlled by the BDMPSZ mechanism, while for larger frequencies it is described by $\mathrm{N}=1$ term in the GLV opacity expansion. We estimate corresponding quenching rates for different values of the heavy quark path length and different $m / E$ ratios.
\end{abstract}

\section{Introduction}

The energy loss of heavy quarks propagating through the media was widely discussed in recent years in different formalisms. In particular the heavy quark energy losses were intensively studied in the BDMPSZ [1-6] approach, starting from [7].

The authors of [7] assumed that like in the vacuum, the heavy quark radiation is suppressed by the dead cone effect,

$\omega \frac{d I^{\mathrm{vac}}}{d \omega d k_{t}^{2}} \sim \frac{\alpha_{s} C_{F}}{\pi^{2}} \frac{k_{t}^{2}}{\left(k_{t}^{2}+\theta^{2} \omega^{2}\right)^{2}}$,

where $I$ is the multiplicity of the heavy quark, $\theta=m / E$ is the dead cone angle, $\mathrm{m}$ is the heavy quark mass, $\omega$ and $\vec{k}_{t}$ are the frequency and the transverse momenta of the radiated gluon, and $E$ is the energy of the heavy quark. They resulting heavy quark quenching rate is then significantly smaller than the one observed in the experiment, where up to rather small energies of order several masses of heavy quark, the jet quenching

a e-mail: blok@physics.technion.ac.il (corresponding author) rates of the heavy and light/massless quarks are the same $[8,9]$.

However it was found in [10,11] (see also [12-15] for related research) that the dead cone effect is actually absent, both in the harmonic oscillator ( $\mathrm{HO})$ approximation to the BDMPSZ approach and in the first $\mathrm{N}=1$ term in the GLV opacity expansion [16-18]. This observation leads to significant increase in the theoretical prediction for the heavy quark jet quenching rate. However this increase is not large enough to match the experimental data on the mass dependence .of the quenching factor. In fact the simulations carried in $[10,11]$ show that in both HO and GLV approximations the quenching rate is approximately constant as a function of a quark mass up to $\theta \sim 0.05$ and then starts to fall.

In another interesting development it was pointed in [10, 19-22] that the interference pattern in the parton propagation is determined by the minimal of the two available coherence lengths - the LPM coherence length, the quantum diffusion formation length and the parton path length $L$. Let us define the quenching coefficient as

$\hat{q}=\int \frac{d^{2} q_{t}}{(2 \pi)^{2}} n q^{2} \frac{d \sigma_{e l}^{R}}{d^{2} q_{t}}$,

where $n$ is the density of the scattering centres in the media, and $\sigma^{R}$ is the scattering cross section of the projectile parton in the color representation $\mathrm{R}$. The transverse momenta accumulated in the diffusion regime is

$k_{t}^{2} \sim \hat{q} l_{c}$,

where $l_{c}$ is a diffusion coherence length, corresponding to Landau Pomeranchuk Migdal (LPM) effect, $l_{c}^{L P M}(\omega)=$ $\sqrt{\omega / \hat{q}}$. Next there is the quantum diffusion formation length, similar to heavy quark propagating in the media without interference between different media scattering centres, $l_{c}^{q} \sim$ $1 /\left(\theta^{2} \omega\right)$. The actual physical radiation regime is determined by the shortest of these lengths $[10,20]$. 
For light quark, as it was shown in [20] for frequencies much smaller than $\omega_{c} \sim \hat{q} L^{2}$, the dynamics of the quark is determined by LPM interference. This interference is usually described in the so called harmonic oscillator (HO) approximation. On the other hand for frequencies $\omega \geq \omega_{c}$, the energy loss is described by the $\mathrm{N}=1 \mathrm{GLV}$ formalism [16-18]. The reason why, although there may be a lot of elastic scatterings, the use of a first term in the opacity expansion is still justified is rather straightforward [20]: the $\mathrm{N}=1 \mathrm{GLV}$ approximation corresponds to the tail of $q_{t}^{2}$ probability distribution, i.e to the regime when the large but rare momentum transfers are dominant.

For heavy quark for the small frequencies the gluons are emitted outside the dead cone and can be described in the same HO approximation as for light quarks. However for frequencies larger than $\omega_{D C}=\left(\hat{q} / \theta^{4}\right)^{1 / 3}$, when the gluons start to be emitted inside the dead cone region, the quantum diffusion length $l_{c}^{q}=1 / \theta^{2} \omega$ starts to be smaller than the diffusion coherence length $l_{c o h}^{L P M}[21,22]$ and the dynamics of radiation for these large frequencies is determined by $\mathrm{N}=$ 1 GLV approximation.

In a further development the authors of [23,24] obtained the formula for light quarks, that explicitly describes not only the diffusion and $\mathrm{N}=1 \mathrm{GLV}$ regime, but also the intermediate region of frequencies, and thus is applicable to the dynamics of light quark quenching in the entire frequency region. Their formula takes into account possible Coulomb interaction corrections to the LPM bremsstrahlung, treated as the perturbation. In the conventional abelian LPM effect such approach is usually called Moller theory [25].

In the current paper we shall generalise the results of $[23,24]$ to heavy quarks and obtain the unified formula that describes the gluon radiation for arbitrary frequencies. The basic approach will be to build the perturbation theory for heavy quark around the HO approximation. We shall see that while the radiation beyond dead cone is determined, for $\omega \leq \omega_{D C}$ by LPM effect and is similar to the one for light quarks, while the dead cone radiation is described by $\mathrm{N}=1$ GLV approximation.

Throughout the whole paper we shall assume that the dominant gluons are soft, $\omega=x E, x<<1$. It is quite simple to include the finite frequencies using $\omega=z(1-z) E$, however the full calculation will then require also the inclusion of the space phase constraints, that will make the calculations much more complicated.

The paper is organised in the following way. In the Sect. 2 we describe the dynamics of heavy quark propagation in the media, in Sect. 3 we review the description of heavy quark in the HO in Sect. 4 we build a perturbation theory for heavy quarks and derive the explicit expression for the energy loss. We use this expression to estimate qualitatively the heavy quark energy loss in Sect. 5, and to estimate the quenching weights in Sect. 6. Our results are summarised in Sect. 7.

\section{Heavy quark propagation in the QGP}

\subsection{Basic formalism}

The heavy quark energy loss in the media is given by

$$
\begin{aligned}
\omega \frac{d I}{d \omega}= & \frac{C_{F} \alpha_{s}}{(\omega)^{2}} 2 \operatorname{Re} \int_{0}^{\infty} d t_{1} \int_{0}^{t_{1}} d t \partial_{\vec{x}} \partial_{\vec{y}}\left(K\left(\vec{x}, t_{1}, \vec{y}, t\right)\right. \\
& \left.-K_{0}\left(\vec{x}, t_{1} ; \vec{y}, t\right)\right)\left.\right|_{\vec{x}=\vec{y}=0 .}
\end{aligned}
$$

Here $K$ is the propagator of the particle in the media with the two dimensional effective potential due. to the scattering centres, and $K_{0}$ is the corresponding propagator of the free particle in the vacuum. The effective two dimensional potential is given by

$V(\vec{\rho})=i \int \frac{d^{2} q_{t}}{(2 \pi)^{2}}\left(1-\exp \left(i \overrightarrow{q_{t}} \vec{\rho}\right)\right) \frac{d^{2} \sigma_{e l}}{d^{2} q_{t}}$.

Here $d^{2} \sigma_{e l} / d^{2} q_{t}$ is the cross section of elastic scattering of high energy particle on the media centre. The media is described by Gyulassy-Wang model [26]. The effective potential in the momentum space is given by

$\frac{d \sigma\left(\vec{q}_{t}\right)}{d^{2} q_{t}}=\frac{4 \pi \alpha_{s} m_{D}^{2} T}{\left(q_{t}^{2}+\mu^{2}\right)^{2}} \equiv \frac{g^{4} n}{\left(q_{t}^{2}+\mu^{2}\right)^{2}}$,

where the parameter $\mu \sim m_{D}$, and the Debye mass $m_{D}$ is given by

$m_{D} \sim 4 \pi \alpha_{s} T^{2}\left(1+N_{f} / 6\right)=\frac{3}{2} g^{2} T^{6}$

for $N_{f}=3$ light quarks, $\mathrm{T}$ is the media/QGP temperature. The density of the scattering centres in the GW model is given by $n=\frac{3}{2} T^{3}$, and the strong coupling is $\alpha_{s}=\frac{g^{2}}{4 \pi}$. The effective potential in the coordinate space is

$V(\rho)=\frac{\hat{q}}{4 N_{c}}\left(1-\mu \rho K_{1}(\mu \rho)=\frac{\hat{q} \rho^{2}}{4 N_{c}}\left(\log \left(\frac{4}{\mu^{2} \rho^{2}}\right)+1-2 \gamma_{E}\right)\right.$,

where $\gamma_{E}=0.577$ is the Euler constant, and the bare quenching coefficient is

$\hat{q}=4 \pi \alpha_{s}^{2} N_{c} n$.

\subsection{Perturbation theory}

For processes that are dominated by large momentum transfer oit is enough to take into account only the first terms in the Taylor expansion of $V(\rho)$. The first approximation corresponds to the quadratic term in the expansion 8 and is 
called the HO (harmonic oscillator) approximation. In this approximation the effective potential $\mathrm{V}$ is given by

$V(\rho)=\frac{1}{4} \hat{q}_{\mathrm{eff}} \rho^{2}$.

Here $\hat{q}_{\text {eff }}$ is the effective jet quenching coefficient, given by

$\hat{q}_{\mathrm{eff}}=\hat{q} \log \left(\frac{Q^{2}}{\mu^{2}}\right)$

and Q is the typical transverse momenta, accumulated by the particle on the scale of the coherence length.

The HO effectively describes the LPM bremsstrahlung [1]. More precise treatment of the energy loss includes also large Coulomb logarithms and is called in the theory of the Abelian (QED) LPM effect the Moller theory [25]. In the QCD framework the inclusion of Coulombic interactions can be made using the perturbation theory $[23,24]$. Namely, instead of the usual opacity expansion [16-18], we shall consider the perturbation theory around the oscillator potential adding the Coulombic effects as a perturbation. The effective potential in Moller theory is given by

$V(\rho)=\frac{1}{4} \hat{q} \rho^{2} \log \left(1 / \rho^{2} \mu^{2}\right)$,

and includes the short range coulombic logarithms. In the framework of the perturbation theory this potential is split as

$$
\begin{aligned}
& V(\rho)=V_{H O}(\rho)+V_{\text {pert }}(\rho), V_{H O}(\rho)=\frac{\hat{q} \log \left(Q^{2} / \mu^{2}\right)}{4} \rho^{2} \\
& V_{\text {pert }}(\rho)=\frac{\hat{q}}{4} \log \left(\frac{1}{Q^{2} \rho^{2}}\right)
\end{aligned}
$$

where $\mathrm{Q}$ is the typical momenta, defined above, equal to $Q \sim$ $\sqrt{\hat{q} \omega}$ in the HO approximation. We shall need sufficiently large $\mathrm{Q}$, so that

$$
\log \left(Q^{2} / \mu^{2}\right) \gg \log \left(\frac{1}{Q^{2} \rho^{2}}\right)
$$

i.e. perturbation theory is applicable meaning that we probe rather small transverse distances.

Then the energy loss is given by Eq. 4, where the propagator $\mathrm{K}$ is calculated in perturbation theory as $[23,24]$

$$
\begin{aligned}
K\left(\vec{x}, t_{1} ; \vec{y}, t\right)= & K_{H O}\left(\vec{x}, t_{1} ; \vec{y}, t\right) \\
& -\int d^{2} z \int_{t}^{t_{1}} d s K_{H O}\left(\vec{x}, t_{1} ; \vec{z}, s\right) V_{\text {pert }}(z) K_{H O}\left(\vec{z}, s ; \vec{y}, t_{1}\right)
\end{aligned}
$$

Here $K_{H O}$ is the heavy quark propagator in the imaginary two dimensional potential $V_{H O}$ [11]:

$$
\begin{aligned}
K_{H O}\left(\vec{x}, t_{1} ; \vec{y}, t\right)= & \frac{i \omega \Omega}{2 \pi \sinh \Omega\left(t_{1}-t\right)} \exp \left(\frac { i \omega \Omega } { 2 } \left\{\operatorname{coth} \Omega\left(t_{1}-t\right)\left(\vec{x}^{2}+\vec{y}^{2}\right)\right.\right. \\
& \left.\left.-\frac{2 \vec{x} \vec{y}}{\sinh \Omega\left(t_{1}-t\right)}\right\}\right) \exp \left(-i \theta^{2} \omega\left(t_{1}-t\right) / 2\right),
\end{aligned}
$$

and

$\Omega=\frac{(1+i)}{2} \sqrt{\hat{q} / \omega}$

In the limit when there is no media this propagator reduces to free quark propagator

$K_{0}\left(\vec{x}, t_{1} ; \vec{y}, t\right)=\frac{i \omega}{2 \pi} \exp \left(i \frac{\omega(\vec{x}-\vec{y})^{2}}{2\left(t_{1}-t\right)}\right)$.

\subsection{Qualitative dynamics of the heavy quark}

The expansion written in the form 15 clearly exhibits the formation lengths described in the Introduction: the heavy quark mass leads to the oscillating exponent $\exp \left(i \theta^{2} \omega / 2\left(t_{1}-t\right)\right)$ in Eq. 16, while the harmonic oscillator part of the propagator 16 oscillates with the frequency $\sqrt{\omega / \hat{q}}$. Then it is clear that when $l_{c}^{q}<<l_{c}^{L P M}$ the oscillations due to heavy quark mass cut off the integral for heavy quark energy loss, the oscillating harmonic oscillator part of the propagator is approximately freezed and the LPM effect is not relevant, the energy loss is defined by the induced radiation on the scattering centresthe $\mathrm{N}=1 \mathrm{GLV}$. On the other hand, in the opposite case, the heavy quark exponent is close to one, and the integral for energy loss is controlled by the HO multiplier. We have LPM bremsstrahlung plus corrections due to coulomb logarithms.

We can now choose the substruction scale $Q$ in the momentum space. As it was explained in $[20,23]$ this scale corresponds to the typical momentum accumulated by the quark along the coherence length propagation. Such momentum squared is $\hat{q} \times \sqrt{\omega / \hat{q}}$ for $\omega<<\omega_{D C}$ and $\sim \theta^{2} \omega^{2} \sim$ $\omega / l_{c}^{q}$ for $\omega>>\omega_{D C}$. Consequently we shall use the interpolation formula

$$
Q^{2}=\sqrt{\omega \hat{q}_{\mathrm{eff}}} U\left(-\omega+\omega_{D C}\right)+\theta^{2} \omega^{2} U\left(\omega-\omega_{D C}\right),
$$

where $U(x)$ is a unit step function: $U(x)=1$ if $x \geq 0$, and $U(x)=0$ if $x \leq 0$. We shall use another interpolation formula to check the sensitivity to the exact $Q$ value in the intermediate region around $\omega_{D C}$ :

$$
Q^{2}=\sqrt{\hat{q}_{\mathrm{ef}} \omega+\theta^{4} \omega^{4}} .
$$


Alternatively, the dynamics of the heavy quark can be approached using the arguments in [20]. Namely, in the LPM (diffusion) regime the distribution over momentum transfers in the scattering on the media centres is described by a gaussian, peaked in the $Q_{t y p}^{2} \sim \sqrt{\hat{q} w}$. The scattering with significantly higher momentum transfers $q_{t}$ is described by the tail of the distribution, which is $\mathrm{N}=1 \mathrm{GLV}$, that essentially describes the independent coulomb scattering on the media centres. In this region the LPM gaussian is parametrically close to zero, and $\mathrm{N}=1 \mathrm{GLV}$ dominates. It was explained in $[21,22]$ that $\mathrm{N}=1$ term in opacity expansion is a good description of large momentun transfer regime, since such scatterings in the tail occur quite rarely. Since inside dead cone the typical momenta is $k_{t}^{2} \sim \omega / l_{c}^{q} \sim \theta^{2} \omega^{2} \gg \sqrt{\hat{q} \omega}$, inside the dead cone we shall find ourselves in the GLV regime.

\section{$2.4 \mathrm{~N}=1 \mathrm{GLV}$}

We shall also need the explicit expression for $\mathrm{N}=1$ term in the opacity expansion for massive quark. The corresponding result was derived in [11], and has the form:

$$
\begin{aligned}
\omega \frac{d I}{d \omega}= & \int d k_{t}^{2} \int_{0}^{\infty} d q^{2} \frac{4 \alpha_{s} C_{F} \hat{q} q k_{t}}{\pi \omega} \frac{L Q_{1}-\sin \left(L Q_{1}\right)}{Q_{1}^{2}} \frac{q^{2}}{q^{2}+\theta^{2} \omega^{2}} \\
& \times \frac{m_{D}^{2}\left(k^{2}+\theta^{2} \omega^{2}\right)+\left(k^{2}-\theta^{2} \omega^{2}\right)\left(k^{2}-q^{2}\right)}{\left(k^{2}+\theta^{2} \omega^{2}\right)\left(\left(m^{2}+k^{2}+q^{2}\right)^{2}-4 k^{2} q^{2}\right)^{3 / 2}} .
\end{aligned}
$$

where

$Q_{1}=\left(q^{2}+\theta^{2} \omega^{2}\right) /(2 * \omega)$.

Here $k_{t}$ is the momentum of the radiated gluon.

\section{Heavy quark in the $\mathrm{HO}$ approximation}

Let us review the leading order contribution to the energy loss of heavy quark, that in our perturbation approach corresponds to Harmonic approximation. There are two parts in the expression 4 due to different regions of integration in $t_{1}$, we shall call them the bulk and the boundary contributions since in one case the integration in $t_{1}$ goes from 0 to $\mathrm{L}$ and in the second from $L$ to $\infty$. Note that the authors of $[23,24]$ used different approach due to results in [19] that permits for massless case the calculation of the integral 4 without splitting into two regions. However, it is not clear how to extend the method of [19] to the case. of massive quarks. We shall review here the heavy quark energy loss calculation in $\mathrm{HO}$ approximation and represent the results in the form of the one dimensional integrals.

\subsection{HO bulk contribution}

This term is equal to

$$
\begin{aligned}
\omega \frac{d I^{\mathrm{HO} \text { Bulk }}}{d \omega}= & \frac{\alpha_{s}}{\omega^{2}} 2 \operatorname{Re} \int_{0}^{L} d t_{1} \int_{0}^{t_{1}} d t \partial_{\vec{x}} \partial_{\vec{y}}\left(K\left(\vec{x}, t_{1} ; \vec{y}, t\right)\right. \\
& \left.-K_{0}\left(\vec{x}, t_{1} ; \vec{y}, t\right)\right)\left.\right|_{\vec{x}=\vec{y}=0}
\end{aligned}
$$

where $K_{0}$ is the propagator of the free heavy quark, and $K_{H O}$ is the heavy quark propagator in the $\mathrm{HO}$ approximation given by Eq. 16 .

After differentiation we obtain in the soft gluon limit:

$$
\begin{aligned}
& \omega \frac{d^{\mathrm{HO} \text { Bulk }}}{d \omega}=-\frac{\alpha_{S} C_{F}}{\pi} \\
& \quad \times 2 \operatorname{Re} \int_{0}^{L} d t_{1} \int_{0}^{t_{1}} d t\left(\frac{\Omega^{2}}{\left(\sinh \left(\Omega\left(t_{1}-t\right)\right)^{2}\right.}-\frac{1}{\left(t_{1}-t\right)^{2}}\right) \\
& \quad \times \exp \left(-i \theta^{2} \omega\left(\left(t_{1}-t\right) / 2\right)\right.
\end{aligned}
$$

Note that the integrand is a function of $\tau=t_{1}-t$. We use the identity

$\int_{0}^{L} d t_{1} \int_{0}^{t_{1}} d s f(s)=\int_{0}^{L}(L-s) f(s) d s$

to go from the double to one-dimensional integrals. This means

$$
\begin{aligned}
\omega \frac{d I^{\mathrm{HO} \text { Bulk }}}{d \omega}= & \frac{-2 \alpha_{s} C_{F}}{\pi} 2 \operatorname{Re} \int_{0}^{L} d t_{1} \\
& \times \int_{0}^{t_{1}} d \tau\left(\frac{\Omega^{2}}{\sinh (\Omega \tau)^{2}}-\frac{1}{\tau^{2}}\right) \operatorname{Exp}\left(-i \tau \theta^{2} \omega / 2\right) \\
= & \frac{-2 \alpha_{s} C_{F}}{\pi} \int_{0}^{L} d s(L-s)\left(\Omega^{2} / \sinh (\Omega s)^{2}-1 / s^{2}\right) \exp \left(-i s \theta^{2} \omega / 2\right)
\end{aligned}
$$

In the limit of the massless quark $\theta \rightarrow 0$ we get the spectrum

$\omega \frac{d I}{d \omega}=\frac{2 \alpha_{s} C_{F}}{\pi} \operatorname{Re} \log \left(\frac{\sinh (\Omega L)}{\Omega L}\right)$

in agreement with the BDMPSZ results for the bulk part of the spectrum for massless quark.

\subsection{HO boundary term}

It is also easy to calculate the boundary term in the $\mathrm{HO}$ approximation:

$$
\begin{aligned}
\omega \frac{d I^{\text {HO Boundary }}}{d \omega}= & \frac{\alpha_{s}}{\omega^{2}} 2 \operatorname{Re} \int_{L}^{\infty} d t_{1} \int_{0}^{L} d t \partial_{\vec{x}} \partial_{\vec{y}}\left(K\left(\vec{x}, t ; \vec{y}, t_{1}\right)\right. \\
& -\left.K_{0}\left(\vec{x}, t_{1} ; \vec{y}, t\right)\right|_{\vec{x}=\vec{y}=0}
\end{aligned}
$$

The propagator $\mathrm{K}$ in Eq. 28 corresponds to the new regime when the particle travels outside of the media, $t_{1}>L, L>$ $t>0$. Consequently it is given by by the convolution 
$K\left(\vec{x}, t_{1} ; \vec{y}, t\right)=\int d^{2} z K_{0}\left(\vec{x}, t_{1} ; \vec{z}, L\right) K_{H O}(\vec{z}, L ; \vec{y}, t)$

Using the explicit expressions for $K_{0}$ and $K_{H O}$ given by Eqs. 16,18 we obtain

$$
\begin{aligned}
& \left.\partial_{\vec{x}} \partial_{\vec{y}} K\left(\vec{x}, t_{1} ; \vec{y}, t\right)\right|_{\vec{x}=0, \vec{y}=0} \\
& =\int d^{2} z \int_{L}^{\infty} d t_{1} \int_{0}^{L} d t \frac{1}{(2 \pi)^{2}} \frac{\omega^{4} \Omega^{2} z^{2}}{\left(t_{1}-L\right)^{2} \sinh (\Omega(L-t))^{2}} \\
& \quad \times \exp \left(i z^{2}\left(\frac{\omega}{2\left(t_{1}-L\right)}+\frac{\omega \Omega}{2} \operatorname{coth} \Omega(L-t)\right)\right) \exp \left(-i \theta^{2} \omega\left(t_{1}-t\right) / 2\right)
\end{aligned}
$$

It is easy to carry the integration over $d^{2} z$. We have

$$
\int d^{2} z \exp \left(i A z^{2}\right) z^{2}=\frac{\pi}{A^{2}}
$$

So we obtain

$$
\begin{gathered}
\omega \frac{d I^{\mathrm{HO}} \text { Boundary }}{d \omega}=\int_{L}^{\infty} d t_{1} \int_{0}^{L} d t\left(2 \operatorname{Re} \frac{1}{\pi} \frac{\Omega^{2}}{\left(t_{1}-L\right)^{2} \sinh \left(\Omega(L-t)^{2}\right.} \frac{\exp \left(-i \theta^{2} \omega\left(t_{1}-L+L-t\right) / 2\right)}{\left(1 /\left(t_{1}-L\right)+\Omega \operatorname{coth} \Omega(L-t)\right)^{2}}\right. \\
\left.-2 \operatorname{Re} \frac{1}{\pi} \frac{\exp \left(-i \theta^{2} \omega\left(t_{1}-L+L-t\right) / 2\right)}{\left(t_{1}-t\right)^{2}}\right)
\end{gathered}
$$

we now can define $s=t_{1}-L$, and take integral over s.

Using the formula

$\int_{0}^{\infty} d s \exp (-i A s) /(1+B s)^{2}=\frac{B-i A \exp (A / B) * \Gamma(0, i A / B))}{B^{2}}$

where $\Gamma(s, x)$ is the incomplete gamma function [27]. We obtain

$$
\begin{aligned}
& \omega \frac{d I^{\mathrm{HO}} \text { Boundary }}{d \omega}=2 \operatorname{Re} \frac{\alpha_{s} C_{F}}{\pi} \\
& \quad \times \int_{0}^{L} d s\left(\frac{2 \Omega}{\sinh (2 \Omega s)}-\frac{i \theta^{2} \omega}{2} \exp \left(i \frac{\theta^{2} \omega \tanh (\Omega s)}{2 \Omega}\right)\right) \\
& \quad \times \frac{\Gamma\left(0, \frac{i \theta^{2} \omega \tanh (\Omega s)}{2 \Omega}\right)}{\cosh (\Omega s)^{2}} \\
& \quad-\left(\frac{1}{s}-\frac{i \theta^{2} \omega}{2} \exp \left(i \theta^{2} \omega s / 2\right) \Gamma\left(0, i \theta^{2} \omega s / 2\right)\right)
\end{aligned}
$$

In Eq. 34

$\Gamma(0, x)=-E i(-x)-i \pi$

and $s=L-t$, the function Ei is the integral exponent function [27]. Note that. the integrand in 34 is concentrated near the end of the media region, i.e. near $t \sim L$.
For small frequencies outside the dead cone the energy spectrum almost does not change when we take into account the quark mass, while for large frequencies in the dead angle region the spectrum decreases rather rapidly, in agreement with Dokshitzer-Kharzeev results.

The full $\mathrm{HO}$ result for massive quarks is then given by the sum of Eqs. 34 and 26:

$\omega \frac{d I^{\mathrm{HO}}}{d \omega}=\omega \frac{d I^{\mathrm{HO} \text { Bulk }}}{d \omega}+\omega \frac{d I^{\mathrm{HO}} \text { Boundary }}{d \omega}$

For light quark (i.e. in the $\theta \rightarrow 0$ limit) we have for the bulk term

$\omega \frac{d I}{d \omega}=\frac{\alpha_{S} C_{F}}{\pi} \operatorname{Re} \log \left(\frac{\sinh (\Omega L)}{\Omega L}\right)$ $\omega \frac{d I}{d \omega}=\frac{\alpha_{s} C_{F}}{\pi}\left(R e \log (|\cosh (\Omega L)|)-R e \log \left(\frac{\sinh (\Omega L)}{\Omega L}\right)\right.$

for the boundary term. In the sum we obtain the famous BDMPS spectrum

$\omega \frac{d I}{d \omega}=\frac{2 \alpha_{s} C_{F}}{\pi}(\log (|\cosh (\Omega L)|)$

confirming the self consistency of our approach.

\section{Coulombic correction}

We are now in position to calculate the corrections due to Coulomb logarithms. As in the previous section we split the integration in Eq. 4 into two parts $0<t_{1}<L$ (the bulk term) and $t_{1}>L$-the boundary term. 


\subsection{Bulk term}

We start from the bulk term. The Coulombic correction to the propagator for the heavy quark is given by [23]

$$
\begin{aligned}
& K_{\text {pert }}\left(\vec{x}, t_{1} ; \vec{y}, t\right)=-\int d^{2} z \int_{0}^{L} d t_{1} \int_{0}^{t_{1}} d t \\
& \quad \times \int_{t}^{t_{1}} d s K_{H O}\left(\vec{x}, t_{1} ; \vec{z}, s\right) V_{\text {pert }}(\vec{z}, s) K_{H O}(\vec{z}, s, ; \vec{y}, t)
\end{aligned}
$$

where the perturbation potential is taken as in Eq. 13

$V_{\text {pert }}=\frac{\hat{q}}{4} \log 1 /\left(z^{2} Q^{2}\right)$

where $\mathrm{Q}$ is the substraction point in momentum space, that must be taken as the typical momentum acquired in the set of elastic scatterings over the coherence length scale. Note that the potential is not dependent on $s$. As a result we have after differentiating the propagator over its endpoints,

$$
\begin{aligned}
& \omega \frac{d I^{\text {Bulk Coulomb }}}{d \omega}=\frac{\alpha_{s} C_{F}}{\omega^{2}} 2 \operatorname{Re} \int_{0}^{L} d t_{1} \int_{0}^{t_{1}} d t \int d^{2} z \int_{t}^{t_{1}} d s \\
& \times \frac{\hat{q}}{4} \frac{\omega^{4} \Omega^{4} \exp \left(-i \theta^{2} \omega\left(t_{1}-t\right) / 2\right)}{(2 \pi)^{2} \sinh \Omega\left(t_{1}-s\right)^{2} \sinh \Omega(s-t)^{2}} \\
& \times \exp \left(\frac{i \omega \Omega}{2} z^{2}\left(\operatorname{coth}\left(\Omega\left(t_{1}-s\right)+\operatorname{coth} \Omega(s-t)\right)\right) z^{4} \log \left(\frac{1}{z^{2} Q^{2}}\right)\right.
\end{aligned}
$$

We now carry the integration over $d^{2} z$ using

$$
\begin{aligned}
& \int d^{2} z z^{4} \log \left(\frac{1}{z^{2} Q^{2}}\right) \exp \left(-B z^{2}\right) \\
& =\frac{\left(-3+2 \gamma_{E}+2 \log \left(\frac{B}{Q^{2}}\right)\right) \pi}{B^{3}}, \operatorname{Re}(B) \geq 0 .
\end{aligned}
$$

We then obtain

$$
\begin{aligned}
& \omega \frac{d I^{\text {Bulk Coulomb }}}{d \omega}=-\frac{4 \hat{q} \alpha_{s} C_{F} \Omega}{\pi \omega} 2 R e \\
& \quad \times \int_{0}^{L} d t_{1} \int_{0}^{t_{1}} d t \int_{t}^{t_{1}} d s \sinh \Omega\left(t_{1}-s\right) \sinh \Omega(s-t) \\
& \quad \times\left(i\left(-3+2 \gamma+2 \log \left(\frac{\omega \Omega}{2 Q^{2}} \frac{\sinh \Omega\left(t_{1}-t\right)}{\left.\sinh \Omega\left(t_{1}-s\right) \sinh \Omega \cdot(s-t)\right]}\right)\right)+\pi\right) \\
& \quad \times \frac{\exp \left(-i \theta^{2} \omega\left(t_{1}-t\right) / 2\right.}{\sinh \left(\Omega\left(t_{1}-t\right)\right)^{3}} .
\end{aligned}
$$

The integral over $s$ can be taken analytically in the limits between $t_{1}, t$. Quite remarkably under regularisation when we integrate between $t+\epsilon, t_{1}-\epsilon$, and then take the limit $\epsilon \rightarrow 0$ the integral is finite. We then use the identity Eq. 25, since the integrand depends only on the difference $t_{1}-t$, to represent the Coulomb correction to the bulk contribution in the form of an one dimensional integral

$$
\begin{aligned}
\omega & \frac{d I^{\text {Bulk Coulomb }}}{d \omega}=\operatorname{Re} \int_{0}^{L} d x(L-x) \\
& \times 2 i \frac{\alpha_{s} C_{F} \hat{q}}{\omega \pi} \frac{\exp \left(i \theta^{2} \omega(-i \theta * \omega x / 2)\right.}{\sinh \Omega(x)^{3}} \\
& \times \cosh (\Omega x)\left(-\left(\left(-2+A+\log (4)-2 \log \left(\Omega * \omega / Q^{2}\right)\right)\right.\right. \\
& +2 *(\Omega x+\log (1-\operatorname{Exp}(-2 \Omega x))-\log (2))) \tanh [\Omega x) \\
& -\left(-\pi^{2} / 6-(2+A) \Omega x-2 \operatorname{Li} 2(2, \exp (-2 \Omega x))\right. \\
& -L i_{2}(2,1)-(-i \pi+2 \Omega x)^{2} / 2-\pi^{2} / 3+2 \Omega x(-i * \pi \\
& +\log \left((1-\exp (-2 \Omega x))+2 \Omega x+\log \left(-\Omega \omega / Q^{2}\right)\right), \quad(45)
\end{aligned}
$$

where

$A=-i \pi+3-2 \gamma_{E}$.

Here $L i_{2}$ is the dilogarithm (Spence) function [27]:

$L_{2}(z)=-\int_{0}^{z} \frac{\log (1-u)}{u}$

Note that, since $\Omega$ is complex, the integrand is the complicated analytical function of its arguments. We have checked that this function has no discontinuities related to the cuts of logarithm and dilogarithm in the complex plane in the region of integration and as a function of $\omega$ and $\hat{q}$.

\subsection{The correction to the boundary term}

Next we need to calculate the correction to the HO boundary term. This correction is given by the integral

$$
\begin{aligned}
& \omega \frac{d I^{\text {Coulomb Boundary }}}{d \omega}=\frac{\alpha_{s} C_{F}}{(2 \pi)^{3} \omega^{2}} \frac{q}{4} \\
& \times \int_{L}^{\infty} d t_{1} \int_{0}^{L} d t \int_{t}^{L} d s \int d^{2} z \int d^{2} u \frac{\exp \left(i \frac{\omega z^{2}}{t_{1}-L}\right)}{\left(t_{1}-L\right)^{2}}(\vec{z} \vec{u}) \\
& \times \exp \left(i \frac{\omega \Omega\left(z^{2} * \operatorname{coth}(L-s)+u^{2} * \operatorname{coth}(L-s)-\frac{2 \vec{z} \vec{u}}{\sinh (L-s)}\right)}{2}\right) \\
& \times \frac{\exp \left(i u^{2} \operatorname{coth}(s-t) \frac{\omega \Omega}{2}\right)}{\sinh \Omega(L-s)(\sinh \Omega(s-t))^{2}} \omega^{5} \Omega^{3} u^{2} \log \left(\frac{1}{Q^{2} u^{2}}\right)
\end{aligned}
$$

The integral $\int d^{2} z$ is gaussian and can be easily taken, the remaining integral over $\int d^{2} u$ is taken using Eq. 43 .

After the integrations over $d^{2} z$ and $d^{2} u$ the resulting integral has the form 


$$
\begin{aligned}
& \omega \frac{d I^{\text {Coulomb Boundary }}}{d \omega}=\int_{L}^{\infty} d t_{1} \int_{0}^{L} d t \int_{t}^{L} d s \frac{i \alpha_{s} C_{F} \hat{q} \Omega}{\pi \omega} \exp \left(i \theta^{2} \omega\left(t-t_{1}\right) / 2\right) \\
& \times\left(A-2 \log \left(\frac{\omega \Omega\left(\cosh (\Omega(L-t)) \Omega\left(t_{1}-L\right)+\sinh (\Omega(L-t))\right.}{2 Q^{2} \sinh (\Omega(s-t))\left(\Omega\left(t_{1}-L\right) \cosh (\Omega(L-s))+\sinh (\Omega(L-s))\right)}\right)\right) \\
& \times \sinh (\Omega(s-t))\left(\Omega\left(t_{1}-L\right) \cosh (\Omega(L-s))+\sinh (\Omega(L-s))\right),
\end{aligned}
$$

where

$$
A=-i \pi+3-2 \gamma_{E} .
$$

The integral over s can be taken using Mathematica, since the integrand is essentially the rational function. We then obtain the double integral over $t, t_{1}$ :

$$
\begin{aligned}
& \omega \frac{d I^{\text {Coulomb Boundary }}}{d \omega}=\frac{i \alpha_{s} C_{F} \hat{q}}{2 \pi \omega} \exp i \theta^{2} \omega\left(t-t_{1}\right) \\
& \times \cosh (\Omega(L-t))\left(2 \operatorname { l o g } \left(\left((1-\exp (-2 \Omega(L-t)))+\Omega\left(t_{1}-L\right)\right.\right.\right. \\
& \left.\left.\times(1+\exp (-2 \Omega(L-t)))) /\left(2 \Omega\left(t_{1}-L\right)\right)\right)+2 \Omega(L-t)\right) \Omega\left(L-t_{1}\right) \\
& -\left(\left(-2+A+\log (4)-2 \log \left(\frac{\Omega \omega}{Q^{2}}\right)\right)+2(\Omega(L-t)\right. \\
& +\log ((1-\exp (-2 \Omega(L-t))))-\log (2)))) \tanh (\Omega(L-t)) \\
& +\left(-\pi^{2} / 6-(2+A) \Omega(L-t)-L i_{2}\left(\frac{\exp (-2 \Omega(L-t))\left(1+\Omega\left(L-t_{1}\right)\right)}{1-\Omega(L-t)}\right.\right. \\
& -L i_{2}\left(\exp (-2 \Omega(L-t))-L i_{2}\left(-1+\frac{2}{\left.1+\Omega\left(L-t_{1}\right)\right)}\right)\right. \\
& -(-i \pi+2 \Omega(L-t))^{2} / 2-(-i \pi+2 \Omega(L-t) \\
& +\log \left(\frac{1+\Omega\left(t_{1}-L\right)}{1+\Omega\left(L-t_{1}\right)}\right)^{2} / 2-\pi^{2} / 3 \\
& +2 \Omega(L-t)\left(\operatorname { l o g } \left(\left(\exp \left(-2 \Omega(L-t)+\frac{-1+\Omega\left(L-t_{1}\right)}{1+\Omega\left(L-t_{1}\right)}\right)\right.\right.\right. \\
& \left.\left.+2 \Omega(L-t)+\log \left(-\frac{\Omega \omega}{Q^{2}}\right)\right)\right) \\
& \left.\times\left(-1+\Omega\left(L-t_{1}\right) \tanh (\Omega(L-t))\right)\right) /\left(\Omega\left(t_{1}-L\right) \cosh (\Omega(L-t))\right. \\
& +\sinh \Omega(L-t))^{3} .
\end{aligned}
$$

The final answer for the coulombic correction is then given by a sum of Eqs. 45,51 :

$\omega \frac{d I^{\text {Coulomb }}}{d \omega}=\omega \frac{d I^{\text {Bulk Coulomb }}}{d \omega}+\omega \frac{d I^{\text {Coulomb Boundary }}}{d \omega}$.

We shall also need $\omega \frac{d I^{\text {Coulomb reduced }}}{d \omega}$ which is given by Eq. 52 but without the common factor $\hat{q}$. The final answer for the energy loss is the sum of Eqs. 52, 36

$$
\begin{aligned}
\omega \frac{d I}{d \omega}(\omega, L, \hat{q}, Q)= & \omega \frac{d I^{\mathrm{HO}}}{d \omega}\left(\omega, L, \hat{q}_{\mathrm{eff}}, Q\right) \\
& +\hat{q} \omega \frac{d I^{\text {Coulomb reduced }}}{d \omega}\left(\omega, L, \hat{q}_{e f f}, Q\right) .
\end{aligned}
$$

where $\hat{q}_{\text {eff }}$ is given by Eq. 11 and the typical momenta $Q$ is given by Eqs. 19, 20. Equation 53 is our main result.
The integrals 45, 51 are rather complicated. However we checked numerically that in the limit of massless quarks $\theta \rightarrow$ 0 our results coincide with the ones obtained in [23,24]. We were not able to reduce the expressions above to the light quark case analytically. However we checked that the zero mass quark expression derived in [23]

$\omega \frac{d I^{\text {Coulomb }}}{d \omega}=\int_{0}^{L} d s \frac{1}{k(s)} \log (k(s)+\gamma)$,

where

$k(s)=\frac{i \omega \Omega}{2}(\operatorname{coth}(\Omega(s)+\tanh (\Omega(L-s))$

coincides with Eq. 53 numerically for all possible values of $L, \omega$

. We have also checked that the expression 55 can be easily derived also summing bulk and boundary contributions, instead of using the approach of [19].

\section{Numerical results}

In our calculations we use our final expression for $\mathrm{HO}+$ Coulomb correction to energy loss - Eqs. 53. For illustrative numerical estimates we take the same parameters as in [24]: $T=0.4 \mathrm{GeV}, \alpha_{s}=0.3$, leading to $\mu=m_{D}=0.9 \mathrm{GeV}$ and $\bar{q} \sim 0.3 \mathrm{GeV}^{3}$. We carried the numerical calculations for two interpolating formula for typical momentum:

$$
Q^{2}=\sqrt{\omega \hat{q}_{\mathrm{eff}}} U\left(-\omega+\omega_{D C}\right)+\theta^{2} \omega^{2} U\left(\omega-\omega_{D C}\right)+\mu^{2},
$$

$Q^{2}=\sqrt{\hat{q}_{\mathrm{ef}} \omega+\theta^{4} \omega^{4}}+\mu^{2}$,

which differ from Eqs. 19, 20 by adding the regularising momenta $\mu^{2}$, similar to the regularisation in [24].

We present our results for energy loss $\omega d I / d \omega$ for two cases; the medium path width $L=2 \mathrm{fm}$ and large path length $L=5 \mathrm{fm}$. We see first that the different choice of the interpolating formula for typical transverse momenta, 19 or 20 does not influence the result qualitatively, although it may induce some difference at small $\omega$ of order $10-15 \%$.

For large $\omega$ beyond dead cone frequency $\omega_{D C}$ we see very good agreement with $\mathrm{N}=1 \mathrm{GLV}$ approximation, especially 

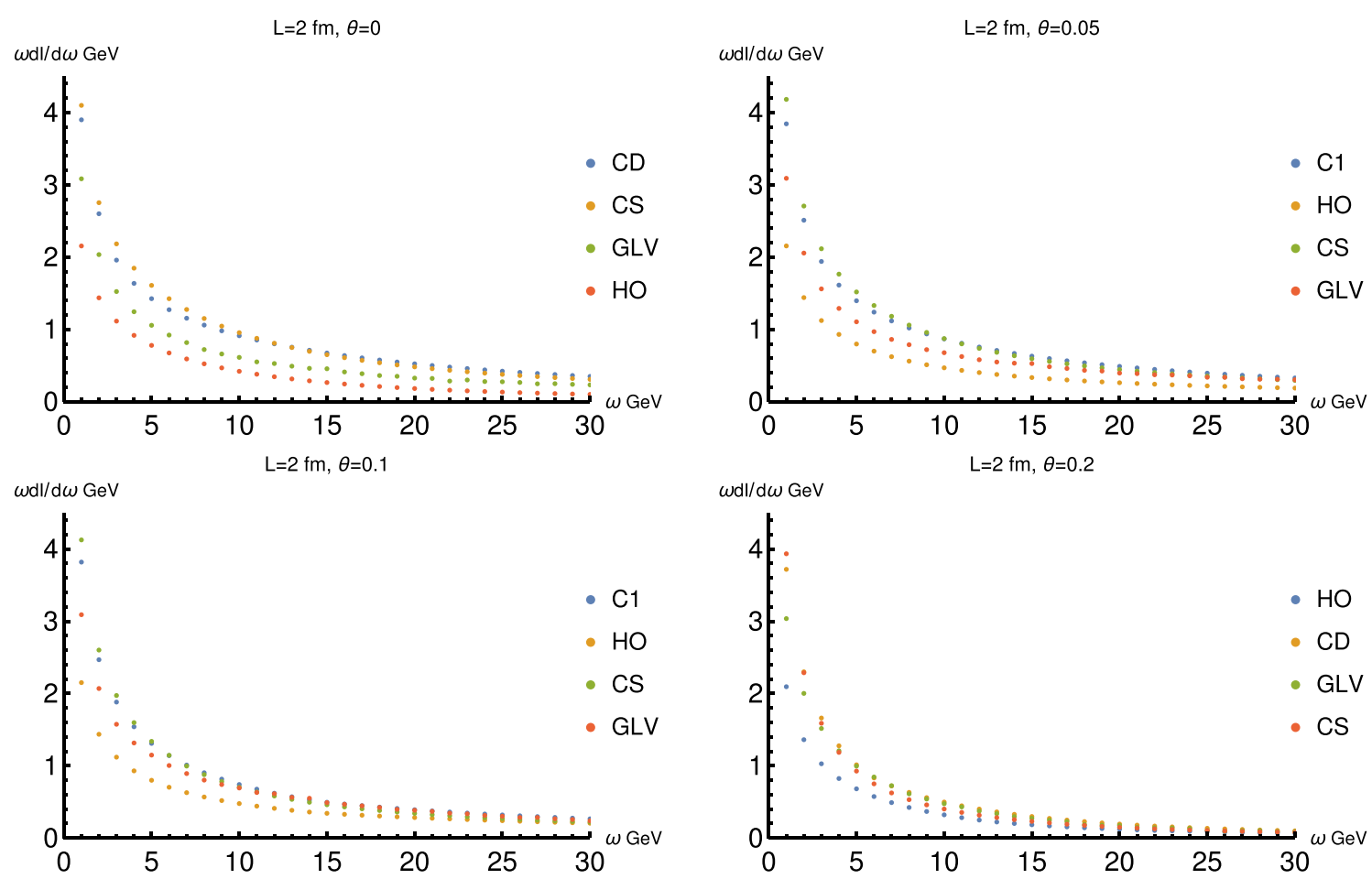

Fig. 1 The energy loss in the leading order in $\alpha_{s}$ for $\mathrm{L}=2 \mathrm{fm}$ for different values of $\theta$ as a function of the radiated gluon energy $\omega$, divided by $\alpha_{S} C_{F}$ for different parametrizations of the substruction momentum $Q(\hat{q}, \omega, \theta)$. We depict the results for harmonic oscillator approximation (HO) and including the Coulomb contribution $(\mathrm{HO}+\mathrm{Coulomb})$ given

by Eq. 53. The curves CD and CS show the dependence of the total energy loss $(\mathrm{HO}+\mathrm{Coulomb})$ given by Eq. 53 on the parametrisation of the substruction momenta Q: CD corresponds to the parametrisation 56, CS to the parametrisation 57, see the discussion in Sect. 2.3. The curve GLV refers to $\mathrm{N}=1 \mathrm{GLV}$ expression with $Q$ independent $\hat{q}$

for the intermediate length case. $\mathrm{L}=2 \mathrm{fm}$. We present $\mathrm{L}=$ $2 \mathrm{fm}$ case in Fig. 1 where we see that the results including coulombic corrections are in very good agreement with $\mathrm{N}=$ $1 \mathrm{GLV}$ for large frequencies beyond dead cone. The use of two interpolating formulae for momenta leads to very close results especially they become identical in the dead cone regime.

In Fig. 2 we depict the similar results for $\mathrm{L}=5 \mathrm{fm}$. We see that in this case for small $\omega$ both $\mathrm{HO}$ and $\mathrm{HO}+$ Coulomb curves lie under the $\mathrm{N}=1 \mathrm{GLV}$ curve. We use Eq. 20 for $Q$. curve, with the agreement increasingly good towards large frequencies corresponding to the region inside dead cone.

In Figs. 3 and 4 we depict for $L=2$ and $5 \mathrm{fm}$ respectively the total and $\mathrm{HO}$ contributions to the energy loss for different values of $\theta$. We see that for the $\theta$ up to 0,05 the energy loss does not change, but for larger $\theta$ it starts to decrease.

\section{Quenching}

Our results for energy loss can be translated to the jet quenching weights along the lines of $[28,29]$. As it is known the jet quenching factor describes the energy loss due to the arbitrary number of Poisson distributed gluons. Indeed, in the

previous chapters we calculated the energy loss probability $\omega d I d \omega$ in the first order in $\alpha_{s}$. Then we can calculate the quenching factor

$$
\begin{aligned}
Q(E) & =\exp \left(-\int_{0}^{\infty}\left(1-\exp \left(-\frac{R}{E} \omega\right) \frac{d I}{d \omega}\right)\right) \\
& =\exp \left(-\frac{R}{E} \int_{0}^{\infty} \exp \left(-\frac{R}{E} N(\omega)\right) \equiv \exp (-S(E))\right.
\end{aligned}
$$

where the multiplicity.

$N(\omega)=\int_{\omega}^{\infty} \omega^{\prime} \frac{d I}{d \omega^{\prime}} d \omega^{\prime}$

and

$R=\frac{d \sigma^{0}}{d p_{t}^{2}}$

is determined from the experimental data, $R \sim 5$. Here $\sigma^{0}$ is the radiation cross section in the vacuum, outside of the media.

The estimated quenching rates have qualitative character, we assumed that for $\omega \leq 1 \mathrm{GeV}$ the $\omega d I / d \omega$ curve linearly goes to zero ar $\omega \rightarrow 0, \omega \leq 1$. We see that for energies 


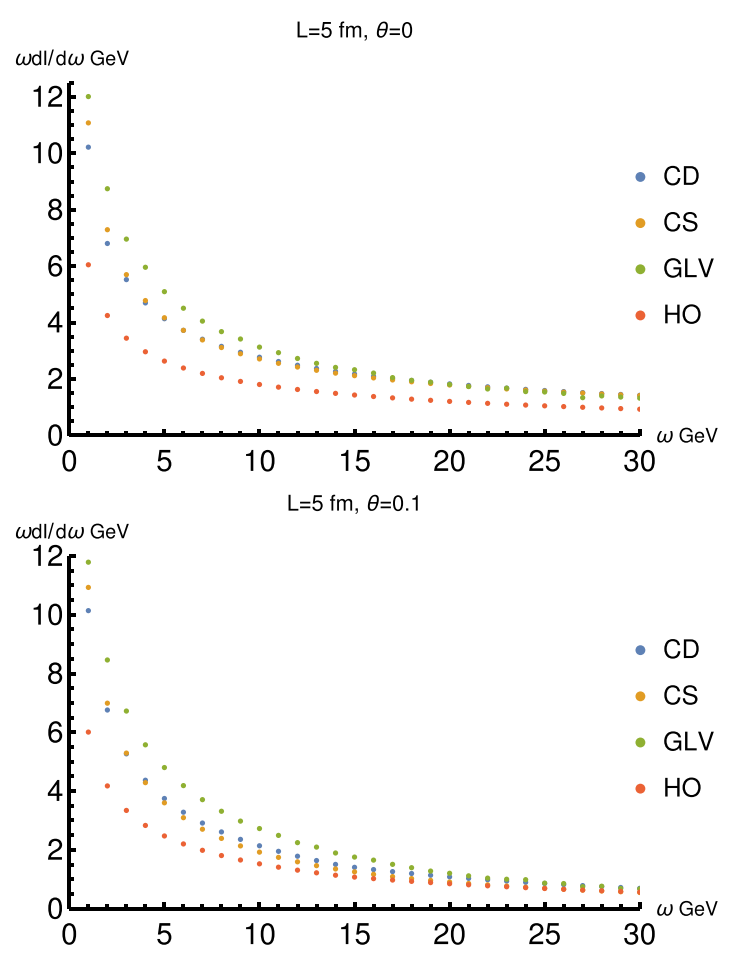

Fig. 2 The energy loss in the leading order in $\alpha_{s}$ for $\mathrm{L}=5 \mathrm{fm}$ for different values of $\theta$ as a function of the radiated gluon energy $\omega$, divided by $\alpha_{S} C_{F}$ for different parameterisations of $Q(\hat{q}, \omega, \theta)$ for $\mathrm{HO}$ and total $(\mathrm{HO}+$ Coulomb $)$ contributions: $\mathrm{CD}, \mathrm{CS}$ are the total $(\mathrm{HO}+\mathrm{Coulomb})$

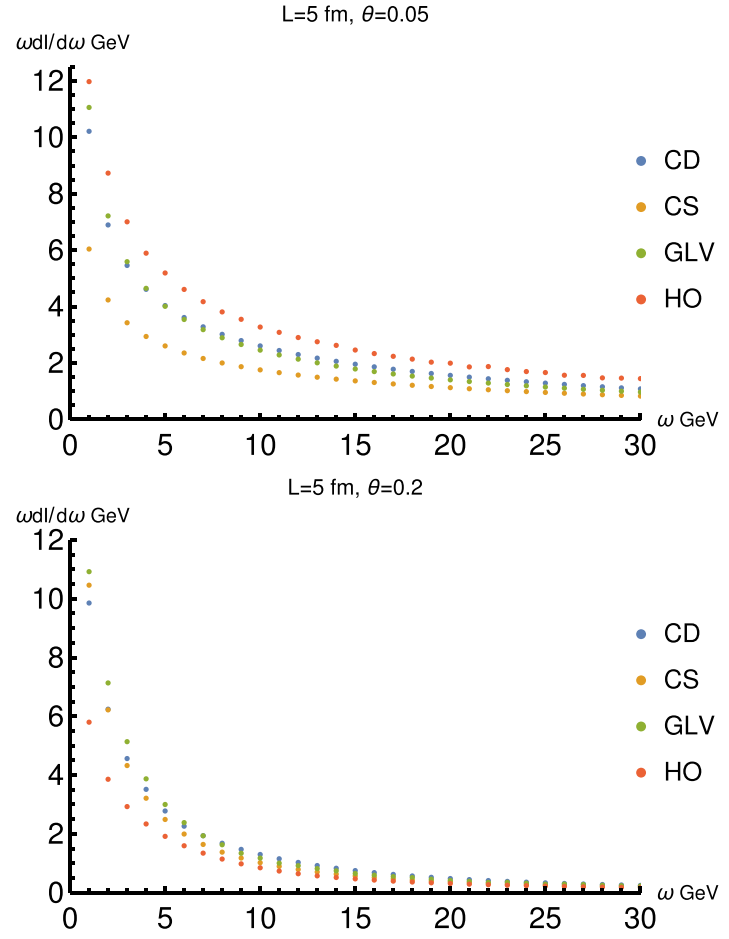

energy losses for typical momenta $Q$ given by Eqs. 19, 20 respectively.HO refers to HO approximation with $Q$ given by Eq. 56, and GLV refers to $\mathrm{N}=1 \mathrm{GLV}$ expression with $Q$ independent $\hat{q}$

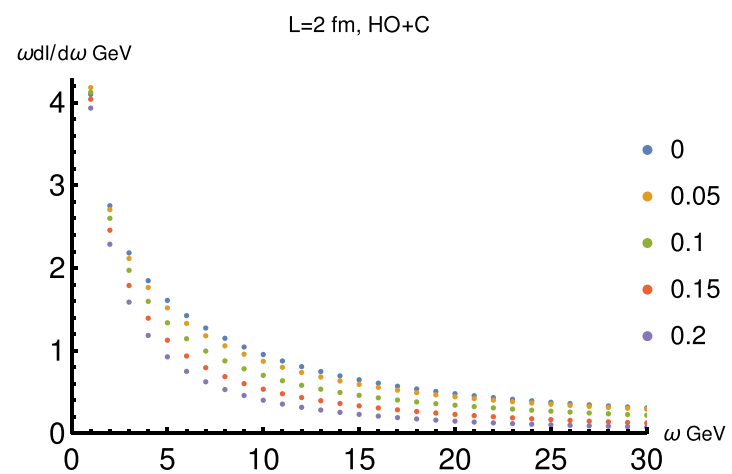

Fig. 3 The energy loss in the leading order in $\alpha_{s}$ for $\mathrm{L}=2 \mathrm{fm}$ for different values of $\theta$ as a function of the radiated gluon energy $\omega$, divided by $\alpha_{s} C_{F}$, right-total energy loss in Moller theory, left-HO approximation. We use Eq. 20 for $Q$

of order $100 \mathrm{GeV}$ (i.e. $\theta \sim 0.05$ the quenching coefficients are actually the same for light quark and heavy b-qiuark. and the quenching coefficients of heavy quarks depend on energy much weaker than for the light, especially for not large L. This is in agreement with the results of [10]. The estimates have very qualitative character especially for light quarks, since we expect they will be further influenced by phase space restrictions which are known to significantly reduce the energy loss, especially for small $\omega$.

\section{Conclusion}

We have calculated the the energy loss of heavy quark propagating through the quark-gluon plasma in the framework of the Moller theory due to the soft gluon emission. In particular we studied the influence of large Coulomb logarithms on the heavy quark propagation. We have found rather large Coulomb corrections to LPM effect for small and large energies of radiated gluons. In particular we have seen that Coulombic corrections lead to N $=1$ GLV expression for energy loss for frequencies corresponding to the 

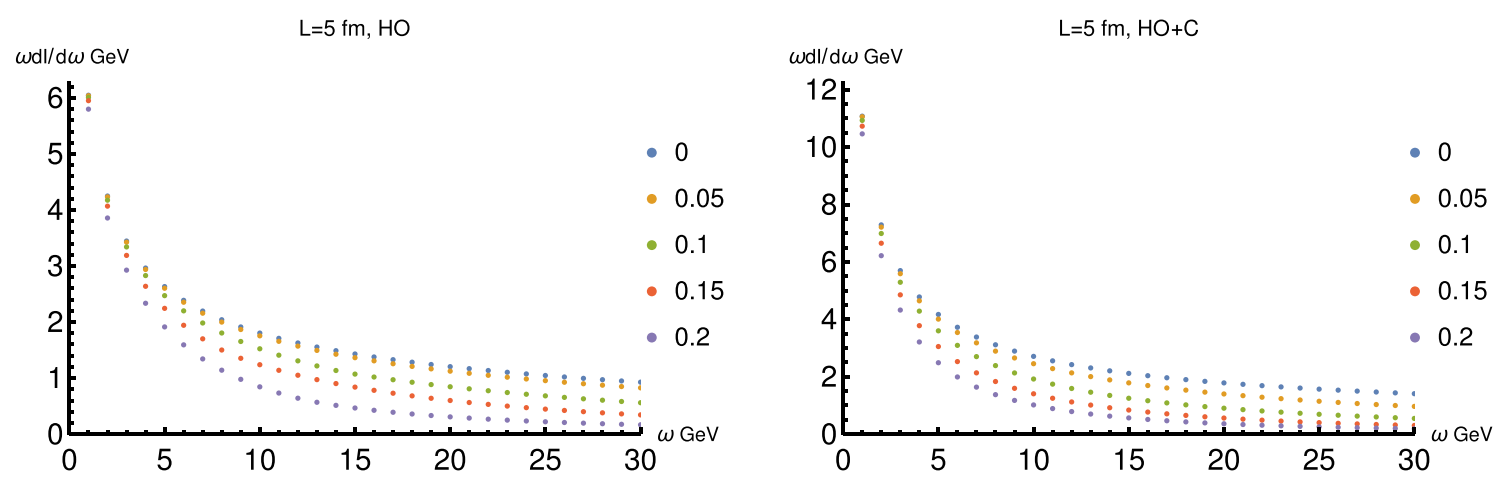

Fig. 4 The energy loss in the leading order in $\alpha_{s}$ for $\mathrm{L}=5 \mathrm{fm}$ for different values of $\theta$ as a function of the radiated gluon energy $\omega$, divided by $\alpha_{S} C_{F}$ right-total energy loss in Moller theory, left-HO approximation.We use Eq. 20 for $Q$

Table 1 The estimate for quenching coefficients $\mathrm{S}(\mathrm{E})$ for light and heavy quarks, for middle $L=2 \mathrm{fm}$ and long $L=5 \mathrm{fm}$ widths. The jet quenching factor $Q(E)=\exp (-S(E))$ Here all $\mathrm{S}(\mathrm{E})$ are divided on $\alpha_{S} C_{F}$

\begin{tabular}{llll}
\hline$L=2 \mathrm{fm}$ & $\begin{array}{l}E=25 \mathrm{GeV} \\
\mathrm{S}(\mathrm{E})\end{array}$ & $\begin{array}{l}E=50 \mathrm{GeV} \\
\mathrm{S}(\mathrm{E})\end{array}$ & $\begin{array}{l}E=100 \mathrm{GeV} \\
\mathrm{S}(\mathrm{E})\end{array}$ \\
\hline Light quark $\mathrm{m}=0$ & 1.74 & 1.38 & 0.83 \\
Heavy quark $m_{b}=5 \mathrm{GeV}$ & 0.75 & 0.79 & 0.77 \\
$L=5 \mathrm{fm}$ & $\mathrm{S}(\mathrm{E})$ & $\mathrm{S}(\mathrm{E})$ & $\mathrm{S}(\mathrm{E})$ \\
Light quark $\mathrm{m}=0$ & 4.74 & 3.84 & 2.5 \\
Heavy quark $m_{b}=5 \mathrm{GeV}$ & 2.55 & 3 & 2.32 \\
\hline
\end{tabular}

radiation inside the dead cone. Our main expression that includes both HO approximation and Coulombic logarithms is an Eq. 53. We have estimated the resulting quenching weights for heavy quark propagation and see that the energy loss of heavy and light quark is approximately the same up to $\theta=m / E \sim 0.05$, We also see that the difference between heavy and light quenching weights decreases with the decrease of the length path of the quark. These results are in agreement with the results of [10]. For massless quarks our results coincide with those of $[23,24]$.

Acknowledgements The author thanks K. Tywoniuk for useful discussions. The work was supported by Israel Science Foundation under the Grant 2025311.

Data Availability Statement This manuscript has no associated data or the data will not be deposited. [Authors' comment: All data generated or analysed during this study are included in this published article.]

Open Access This article is licensed under a Creative Commons Attribution 4.0 International License, which permits use, sharing, adaptation, distribution and reproduction in any medium or format, as long as you give appropriate credit to the original author(s) and the source, provide a link to the Creative Commons licence, and indicate if changes were made. The images or other third party material in this article are included in the article's Creative Commons licence, unless indicated otherwise in a credit line to the material. If material is not included in the article's Creative Commons licence and your intended use is not permitted by statutory regulation or exceeds the permitted use, you will need to obtain permission directly from the copyright holder. To view a copy of this licence, visit http://creativecomm ons.org/licenses/by/4.0/.

Funded by SCOAP $^{3}$.

\section{References}

1. R. Baier, Y.L. Dokshitzer, A.H. Mueller, S. Peigne, D. Schiff, https://doi.org/10.1016/S0550-3213(96)00581-0. arXiv:hep-ph/9608322

2. R. Baier, Y.L. Dokshitzer, A.H. Mueller, S. Peigne, D. Schiff, Nucl. Phys. B 483, 291 (1997). https://doi.org/10.1016/ S0550-3213(96)00553-6. arXiv:hep-ph/9607355

3. R. Baier, Y.L. Dokshitzer, S. Peigne, D. Schiff, Phys. Lett. B 345, 277 (1995). https://doi.org/10.1016/0370-2693(94)01617-L. arXiv:hep-ph/9411409

4. B.G. Zakharov, JETP Lett. 63, 952 (1996). https://doi.org/10.1134/ 1.567126. arXiv:hep-ph/9607440

5. B.G. Zakharov, JETP Lett. 65, 615 (1997). https://doi.org/10.1134/ 1.567389

6. R. Baier, D. Schiff, B.G. Zakharov, Ann. Rev. Nucl. Part. Sci. 50, 37 (2000). https://doi.org/10.1146/annurev.nucl.50.1.37. arXiv:hep-ph/0002198

7. Y.L. Dokshitzer, D.E. Kharzeev, Phys. Lett. B 519, 199 (2001). https://doi.org/10.1016/S0370-2693(01)01130-3. arXiv:hep-ph/0106202

8. T.W. Wang, [CMS Collaboration], Nucl. Part. Phys. Proc. 289-290, 229 (2017). https://doi.org/10.1016/j.nuclphysbps.2017.05.051

9. K. Jung [CMS Collaboration], Nucl. Phys. A 931, 470 (2014). https://doi.org/10.1016/j.nuclphysa.2014.09.086. arXiv: 1410.2576 [nucl-ex]

10. P. Aurenche, B.G. Zakharov, JETP Lett. 90, 237 (2009). https:// doi.org/10.1134/S0021364009160048. arXiv:0907.1918 [hep-ph]

11. N. Armesto, C.A. Salgado, U.A. Wiedemann, Phys. Rev. D 69, 114003 (2004). https://doi.org/10.1103/PhysRevD.69.114003. arXiv:hep-ph/0312106

12. N. Armesto, A. Dainese, C.A. Salgado, U.A. Wiedemann, Phys. Rev. D 71, 054027 (2005). arXiv:hep-ph/0501225

13. M. Djordjevic, M. Gyulassy, Nucl. Phys. A 733, 265 (2004). arXiv:nucl-th/0310076 
14. L. Zhang, D.F. Hou, G.Y. Qin, Phys. Rev. C 100(3), 034907 (2019). https://doi.org/10.1103/PhysRevC.100.034907. arXiv:1812.11048 [hep-ph]

15. B. Blok, K. Tywoniuk, Eur. Phys. J. C 79(7), 560 (2019). https://doi. org/10.1140/epjc/s10052-019-7061-4. arXiv:1901.07864 [hep$\mathrm{ph}]$

16. M. Gyulassy, P. Levai, I. Vitev, Nucl. Phys. B 594, 371 (2001). $\quad$ https://doi.org/10.1016/S0550-3213(00)00652-0. arXiv:nucl-th/0006010

17. U.A. Wiedemann, M. Gyulassy, Nucl. Phys. B 560, 345 (1999). https://doi.org/10.1016/S0550-3213(99)00458-7. arXiv:hep-ph/9906257

18. U.A. Wiedemann, Nucl. Phys. B 588, 303 (2000). https://doi.org/ 10.1016/S0550-3213(00)00457-0. arXiv:hep-ph/0005129

19. P.B. Arnold, Phys. Rev. D 79, 065025 (2009). https://doi.org/10. 1103/PhysRevD.79.065025. arXiv:0808.2767 [hep-ph]

20. P.B. Arnold, Phys. Rev. D 80, 025004 (2009). https://doi.org/10. 1103/PhysRevD.80.025004. arXiv:0903.1081 [nucl-th]

21. B.G. Zakharov, JETP Lett. 73, 49 (2001)

22. B.G. Zakharov, Pisma Zh. Eksp. Teor. Fiz. 73, 55 (2001). arXiv:hep-ph/0012360
23. Y. Mehtar-Tani, JHEP 1907, 057 (2019). https://doi.org/10.1007/ JHEP07(2019)057. arXiv:1903.00506 [hep-ph]

24. Y. Mehtar-Tani, K. Tywoniuk, arXiv:1910.02032 [hep-ph]

25. V.N. Baier, V.M. Katkov, Phys. Rep. 409, 261 (2005). https://doi. org/10.1016/j.physrep.2004.11.003

26. M. Gyulassy, X.n. Wang, Nucl. Phys. B 420, 583 (1994). https:// doi.org/10.1016/0550-3213(94)90079-5. arXiv:nucl-th/9306003

27. M. Abramowitz, I.A. Stegun, Handbook of Mathematical Functions: with Formulas, Graphs, and Mathematical Tables (Dover Publications, New York, 1965)

28. R. Baier, Y.L. Dokshitzer, A.H. Mueller, D. Schiff, JHEP 0109, 033 (2001). https://doi.org/10.1088/1126-6708/2001/09/ 033. arXiv:hep-ph/0106347

29. C.A. Salgado, U.A. Wiedemann, Phys. Rev. D 68, 014008 (2003). https://doi.org/10.1103/PhysRevD.68.014008 